\title{
Enhancer dysfunction: how the main regulators of gene expression contribute to cancer
}

\author{
Siavash K Kurdistani*
}

\begin{abstract}
A genome-wide epigenetic analysis of enhancer elements in colon cancer has implicated distal gene regulatory DNA sequences in the establishment of an oncogenic transcriptional program.
\end{abstract}

What determines the phenotypic identity of a cell is the expression of a particular set of genes in space and time. Such selective gene expression patterns are achieved through highly choreographed interactions between various DNA elements and several multi-protein complexes. The principle DNA elements responsible for cell type-specific gene expression are the enhancer sequences. A recent study [1] now reveals that cancer cells exploit enhancers to reprogram global gene expression in their favor.

\section{Enhancers as distal gene regulatory elements}

Enhancers are short DNA sequences with binding sites for several transcription factors that are largely responsible for specificity of gene expression patterns in a given cell. First discovered through studies of the simian virus 40 (SV40) genome [2], enhancers can remotely regulate transcription from thousands of base pairs away in either direction or orientation relative to their target genes. Although these molecular characteristics define enhancer function, enhancer elements can also be identified by specific features of the chromatin in which they are embedded. These include variations in chromatin compaction, which can be monitored by enzymes that digest the DNA (such as DNase I), and combinations of histone modifications, such as histone 3 lysine 4 monomethylation (H3K4me1), a seemingly ubiquitous marker of enhancers, and more specific acetylation (ac) and

*Correspondence: skurdistani@mednet.ucla.edu

Department of Biological Chemistry and Eli and Edythe Broad Center of

Regenerative Medicine and Stem Cell Research, David Geffen School of Medicine, University of California, Los Angeles, CA 90095, USA methylation of histones $\mathrm{H} 3$ and $\mathrm{H} 4$ [3]. The epigenetic marks are established through recruitment of histonemodifying enzymes by the transcription factors that are bound to the enhancers. As a result, a combinatorial pattern of histone modifications may not only identify enhancers but also provide a measure of the level of enhancer functional activity. For instance, presence of H3K4me1 with H3K27ac probably indicates an active enhancer whose target gene(s) is currently expressed. Loss of H3K4me1 or co-presence of H3K4me1 and a repressive histone mark such as H3K27me3 suggests an inactive enhancer whose target gene is being repressed. How enhancers locate and interact with promoters at a distance remains an area of active investigation but current evidence suggests that architectural looping of chromatin, facilitated in part by cohesin and CCCTCbinding factor (CTCF), is required to bring the enhancers and promoters to close physical proximity of each other. Once juxtaposed, the Mediator co-activator complex coordinates functional interactions between enhancerbound transcription factors and the promoter-bound basal transcription machinery, including RNA polymerase II [4].

\section{Redefining long-distance relationships in cancer}

Previous studies have provided evidence that regions of the genome that are associated with the predisposition to cancer but are far from any annotated gene loci may coincide with enhancers [5]. A comprehensive recent study by Akhtar-Zaidi et al. [1] has now revealed that, in colon cancer, enhancer elements are indeed critical in promoting an oncogenic gene expression program on a cellular scale and are significantly associated with genetic risk variants. Using chromatin immunoprecipitation combined with next-generation sequencing (ChIP-seq), the authors [1] determined the genome-wide distribution of H3K4me1 in normal colon epithelia and nine colorectal cancer (CRC) cell lines that represent different stages of cancer progression, including metastasis. Interestingly, the overall distributions of H3K4me1 in normal colon and cancer cells were more similar to each other than either are to normal or cancer cells derived from other tissue types, indicating that, to some degree, 
cancer cells retain the epigenetic identity of the tissue from which they originally arose. Nonetheless, in cancer cells thousands of regions either gained or lost H3K4me1 when compared with normal colon cells. These regions were termed variant enhancer loci (VELs), a limited number of which were confirmed to indeed have enhancer activity in a standard assay. The prevalence of VELs is even more widespread, as the authors estimate that 40 to $60 \%$ of VELs remain to be discovered [1]. Despite the large number of VELs, only approximately 200 were common to all nine CRC cell lines, indicating a level of heterogeneity in affected enhancers depending on the cancer cell line. The VELs that were lost in colon cancer cells were found to be present most often in normal colon epithelium compared to non-colon normal or cancer cells. In contrast, the gained VELs in CRCs were also found commonly in other cell lines. This is consistent with a model in which colon cancer cells have dedifferentiated from a colon-specific to a more generic phenotype.

The VELs also showed other epigenetic changes, including acetylation and deacetylation of H3K27 in a large fraction of gained and lost VELs, respectively, as well as altered sensitivity to DNase I. These chromatin alterations suggest changes in enhancer activity and expression of the associated gene(s). To determine whether this is the case, the authors [1] had first to determine which VELs are associated with which genes - not a trivial task considering the molecular characteristics of enhancers. Taking multiple bioinformatics approaches and performing statistical gymnastics, they developed an effective algorithm for assigning VELs to specific genes, which takes into account, among other information, gene expression levels and the assumption that CTCF binding sites block enhancer-promoter interactions. Using the algorithm, the authors [1] found that the genes that were linked to gained VELs were expressed at higher levels in CRCs than normal cells and also gained H3K27ac and became hypersensitive to DNase I. The converse was true for genes with lost VELs. And the more VELs were linked to a gene, the more pronounced was the gene expression difference between normal and CRC cells. The VELlinked genes were also more likely to be aberrantly expressed in primary tumors than were genes not linked to VELs. It is conceivable that the expression of the VELlinked genes could also serve as a prognostic signature, but this was not tested in the report [1].

To provide further evidence that the VELs contribute to CRC development and/or progression, the authors [1] showed that the VELs, especially those that are turned off in CRC cells, are significantly associated with single nucleotide polymorphisms (SNPs) that have been shown through genome-wide association studies to confer increased risk of developing CRC. In some instances, the
SNPs overlapped physically with VELs. The SNPs may affect binding of transcription factors to the enhancers and modulate the enhancers' effects on their target gene expression. Taking all the data together, the evidence supports the view that improper usage of enhancer elements, especially the silencing of tissue-specific enhancers, can promote a carcinogenic gene expression program.

It will be interesting to determine how epigenetic changes at enhancers are achieved in cancer cells. These epigenetic changes indicate alterations in targeting of transcription factors and cofactors that establish the epigenetic marks at enhancers in the first place. One important factor is the p300 lysine acetyltransferase (KAT), the binding of which has also been shown to predict enhancer sequences $[3,6]$. The p300 KAT and its closely related paralog CBP acetylate H3K27 (in addition to other lysine residues). So it is possible that redistribution of H3K27ac from lost to gained VELs could at least be partly due to redistribution of p300/CBP KATs. Notably, significant redistribution of p300/CBP with effects on another histone acetylation site implicated in the cancer phenotype, H3K18 $[7,8]$, is also required for virus-mediated oncogenic transformation [9]. Similar relocalization scenarios could apply in principle to other epigenetic enzymes and histone modifications, including H3K4me1, resulting in epigenetic reprogramming of cancer cells. Because histone-modifying enzymes generally do not bind directly to DNA, their redistribution would depend on their interactions with the sequence-specific DNA binding proteins that recruit them. Intriguingly, mutations in epigenetic enzymes, including p300 and CBP, that could modify their enzymatic activities or interactions with other proteins are increasingly reported in cancer [10]. As Akhtar-Zaidi et al. [1] suggest, the VELs may in fact represent the culmination of the complex interactions between genetic and epigenetic elements that redefine which enhancers are turned on or off in cancer.

\section{Competing interests}

The author declares that he has no competing interests.

\section{Acknowledgements}

I thank Michael Carey for scientific input.

\section{Published: 23 May 2012}

\section{References}

1. Akhtar-Zaidi B, Cowper-Sal Lari R, Corradin O, Saiakhova A, Bartels CF, Balasubramanian D, Myeroff L, Lutterbaugh J, Jarrar A, Kalady MF, Willis J, Moore JH, Tesar PJ, Laframboise T, Markowitz S, Lupien M, Scacheri PC: Epigenomic enhancer profiling defines a signature of colon cancer. Science 2012. doi: 10.1126/science.1217277.

2. Müller MM, Gerster T, Schaffner W: Enhancer sequences and the regulation of gene transcription. Eur J Biochem 1988, 176:485-495.

3. Heintzman ND, Hon GC, Hawkins RD, Kheradpour P, Stark A, Harp LF, Ye Z, Lee LK, Stuart RK, Ching CW, Ching KA, Antosiewicz-Bourget JE, Liu H, Zhang X, 
Green RD, Lobanenkov VV, Stewart R, Thomson JA, Crawford GE, Kellis M, Ren B: Histone modifications at human enhancers reflect global cell-typespecific gene expression. Nature 2009, 459:108-112.

4. Ong CT, Corces VG: Enhancer function: new insights into the regulation of tissue-specific gene expression. Nat Rev Genet 2011, 12:283-293.

5. Jia L, Landan G, Pomerantz M, Jaschek R, Herman P, Reich D, Yan C, Khalid O, Kantoff P, Oh W, Manak JR, Berman BP, Henderson BE, Frenkel B, Haiman CA, Freedman M, Tanay A, Coetzee GA: Functional enhancers at the gene-poor 8q24 cancer-linked locus. PLoS Genet 2009, 5:e1000597.

6. Visel A, Blow MJ, Li Z, Zhang T, Akiyama JA, Holt A, Plajzer-Frick I, Shoukry M, Wright C, Chen F, Afzal V, Ren B, Rubin EM, Pennacchio LA: ChIP-seq accurately predicts tissue-specific activity of enhancers. Nature 2009, 457:854-858.

7. Seligson DB, Horvath S, McBrian MA, Mah V, Yu H, Tze S, Wang Q, Chia D, Goodglick L, Kurdistani SK: Global levels of histone modifications predict prognosis in different cancers. Am JPathol 2009, 174:1619-1628.

8. Barber MF, Michishita-Kioi E, Xi Y, Tasselli L, Kioi M, Mogtaderi Z, Tennen RI, Paredes S, Young NL, Chen K, Struhl K, Garcia BA, Gozani O, Li W, Chua KF: SIRT7 links H3K18 deacetylation to maintenance of oncogenic transformation. Nature 2012. doi:10.1038/nature1 1043.

9. Ferrari R, Pellegrini M, Horwitz GA, Xie W, Berk AJ, Kurdistani SK: Epigenetic reprogramming by adenovirus E1A. Science 2008, 321:1086-1088.

10. Iyer NG, Ozdag H, Caldas C: p300/CBP and cancer. Oncogene 2004, 23:4225-4231.

doi:10.1186/gb-2012-13-5-156

Cite this article as: Kurdistani SK: Enhancer dysfunction: How the main regulators of gene expression contribute to cancer. Genome Biology 2012, 13:156. 